Projets

de paysage

\section{Projets de paysage}

Revue scientifique sur la conception et l'aménagement de l'espace

$16 \mid 2017$

Arbres et paysages

\title{
Radioscopie des territoires en Afrique à travers l'arbre (Cameroun et Burkina Faso)
}

An In-depth Study of African Regions through Trees (Cameroon and Burkina Faso)

Jean-Louis Yengué

\section{(2) OpenEdition}

\section{Journals}

Édition électronique

URL : http://journals.openedition.org/paysage/6147

DOI : 10.4000/paysage.6147

ISSN : 1969-6124

Éditeur :

École nationale supérieure du paysage de Versailles-Marseille, Institut national des sciences appliquées Centre Val de Loire - École de la nature et du paysage, École nationale supérieure d'architecture et de paysage de Bordeaux, École nationale supérieure d'architecture et de paysage de Lille, Agrocampus Angers

\section{Référence électronique}

Jean-Louis Yengué, « Radioscopie des territoires en Afrique à travers l'arbre (Cameroun et Burkina Faso) », Projets de paysage [En ligne], 16 | 2017, mis en ligne le 12 juillet 2017, consulté le 30 avril 2020. URL : http://journals.openedition.org/paysage/6147 ; DOI : https://doi.org/10.4000/paysage.6147

Ce document a été généré automatiquement le 30 avril 2020

Projets de paysage 


\title{
Radioscopie des territoires en Afrique à travers l'arbre (Cameroun et Burkina Faso)
}

\author{
An In-depth Study of African Regions through Trees (Cameroon and Burkina \\ Faso)
}

Jean-Louis Yengué

1 Le travail présenté porte sur différents territoires africains (figure 1), des bas-fonds humides de Bafoussam (Cameroun) aux savanes sèches de Barani (Burkina Faso), des grandes villes africaines (Yaoundé, Ouagadougou) aux villages les plus reculés (Mokolo, Sampieri). L'apparente diversité de ces terrains cache en fait une forte convergence : la place de l'arbre dans le paysage. Dans cette contribution, nous considérons le paysage comme une production des sociétés. Une communauté, un groupe d'individus, de par ses habitudes, son mode de vie, son contexte socioéconomique, sa culture, produit du paysage. Cela se traduit spatialement par les différents usages du sol, imbriqués et interdépendants (Roger, 1995; Donadieu, 2012; Küster et Marwinski, 2013; Marcel, 2006 ; Lespez et Ballouche, 2009). Ce paysage est une sécrétion, un produit des sociétés avec comme figure emblématique l'arbre. Ce végétal est omniprésent, aussi bien physiquement que dans les cultures locales. Il enrichit les sols, freine l'érosion due au ruissellement, apporte de l'ombre, permet de se chauffer, de se nourrir, de s'éclairer, de se soigner, etc. Il tient également une place importante dans l'imaginaire des populations locales et est un élément essentiel à l'agriculture. Il peut être aussi une source de crispation et de conflit entre les peuples. Dès lors, l'étude du rapport à l'arbre peut servir à une meilleure connaissance des territoires par l'analyse de la dimension spatiale des rapports sociaux. L'objectif de cette contribution est d'en proposer une lecture avec comme entrée le décryptage des usages de l'arbre aussi bien en ville que dans les champs. Les rapports sociaux sont ici entendus comme « des rapports entre des hommes vivant à un moment donné en un lieu déterminé ; rapports du groupe qu'ils constituent avec d'autres groupes» (Herin, 1986). Ces rapports sociaux s'entremêlent avec les objets spatiaux (les arbres par exemple) pour définir une grande 
variété de combinaisons géographiques orchestrées par la vie sociale, son histoire passée et présente (Méo, 2016).

Figure 1. Localisation des sites d'étude

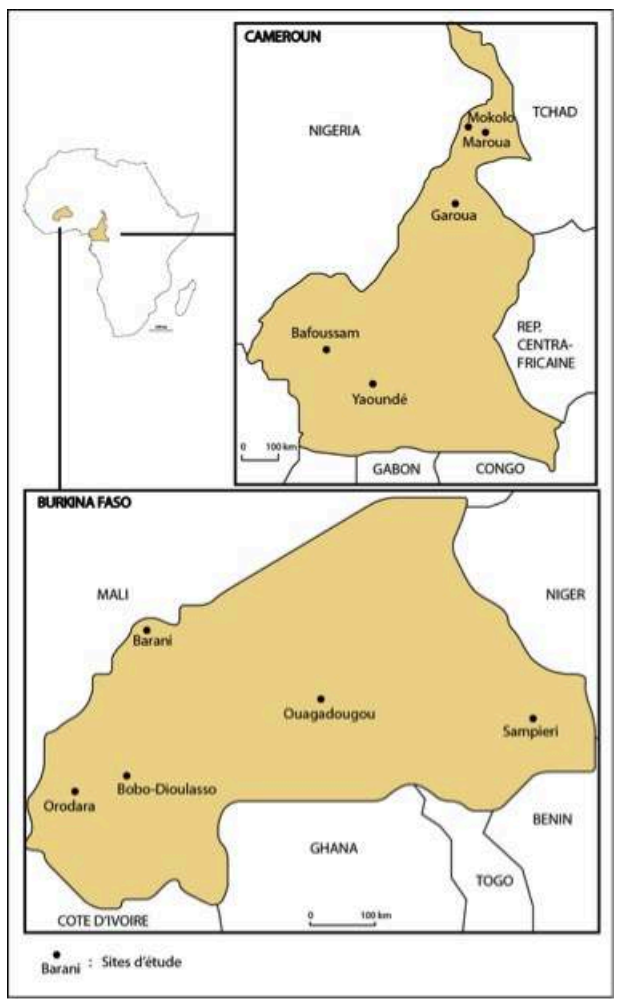

2 Notre démarche conceptuelle s'inscrit dans la phytogéographie (Portères, 1961) que nous plaçons dans le champ de la géographie culturelle (Claval, 1992 ; Méo, 2010). Il s'agit de comprendre les espaces en établissant et en étudiant les liens entre les sociétés et leur environnement, entre les groupes humains et les arbres. Nous l'avons mise en place à la fin des années 1990 (Yengué, 2000) et nous la poursuivons depuis à travers plusieurs programmes de recherche ${ }^{1}$. Le présent article propose une synthèse de quelques-uns des résultats. La méthodologie développée s'appuie sur des outils communs aux sciences sociales : le corpus initial de données est bien souvent textuel et iconographique issu d'entretiens, d'enquêtes, de relevés de terrain. Nous mobilisons aussi l'analyse d'images, la télédétection et les systèmes d'informations géographiques. Si la mise en œuvre diffère, en fonction des terrains, des époques, des équipes, elle présente une constance : l'entretien avec les acteurs du paysage dans le paysage. C'est assez proche des enquêtes participatives (Blanchet et Gotman, 2010 ; Combessie, 2007) qu'Alain Touraine définit comme la compréhension de l'autre dans le partage d'une condition commune (Touraine, 1965). En effet, en participant aux activités des groupes et en se faisant accepter par ses membres, nous pouvons plus facilement comprendre les logiques, les enjeux et la complexité du territoire. Aussi, nous avons parcouru les territoires étudiés (figure 1) en compagnie des acteurs locaux : agriculteurs, chefs locaux, responsables administratifs et employés municipaux, etc.

Nos travaux montrent que le couvert arboré et ses dynamiques sont le produit d'un subtil dosage entre le besoin immédiat de vivre, de survivre et la volonté de préserver les ressources de la terre à long terme (Yengué, 2015). Ce dosage est totalement 
tributaire d'un faisceau d'éléments fortement interdépendants et organisé autour de rapports sociaux entre les populations et ceux qui détiennent le pouvoir, entre les locaux et les étrangers, entre les vieux et les jeunes, entre les hommes et les femmes. Le tout forme un système complexe dont le rapport à l'arbre est l'une des clés de lecture. En ville comme dans les champs, nous montrerons que ce rapport est complexe, différent selon le lieu, l'essence, l'ethnie, et fortement mouvant dans le temps. À travers l'arbre nous exposerons les pratiques agricoles (première partie) et les stratégies de survie (deuxième partie). L'arbre peut également être un objet de compréhension des jeux de pouvoir et de domination (troisième partie) et des dépassements des conflits (quatrième partie).

\section{L'arbre des pratiques agricoles}

Les formations boisées les plus fréquemment rencontrées en Afrique se caractérisent par la présence répétitive de certaines espèces d'arbres qui dominent tout le paysage, une domination d'autant plus évidente qu'elles ne se développent que sur des champs dégagés pour les cultures (figures 2, 3 et 4). Ces formations sont appelées par la communauté scientifique parcs arborés ou parcs agroforestiers (Pélissier, 1980; Yameogo et al., 2013 ; Garine et al., 2013 ; Smektala et al., 2005).

Figure 2. Parc à Vitellaria paradoxa. Région de Barani (Burkina Faso), février 2012

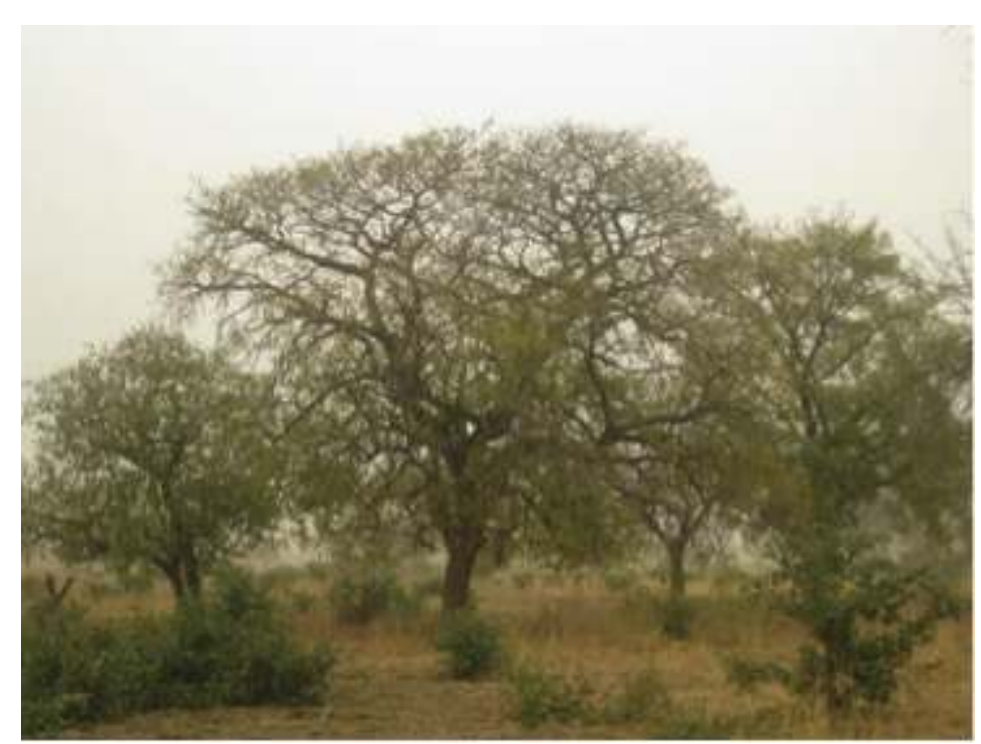


Figure 3. Parc à Faidherbia albida et à Borassum aethiopum. Région de Mokolo (Cameroun), avril 2010

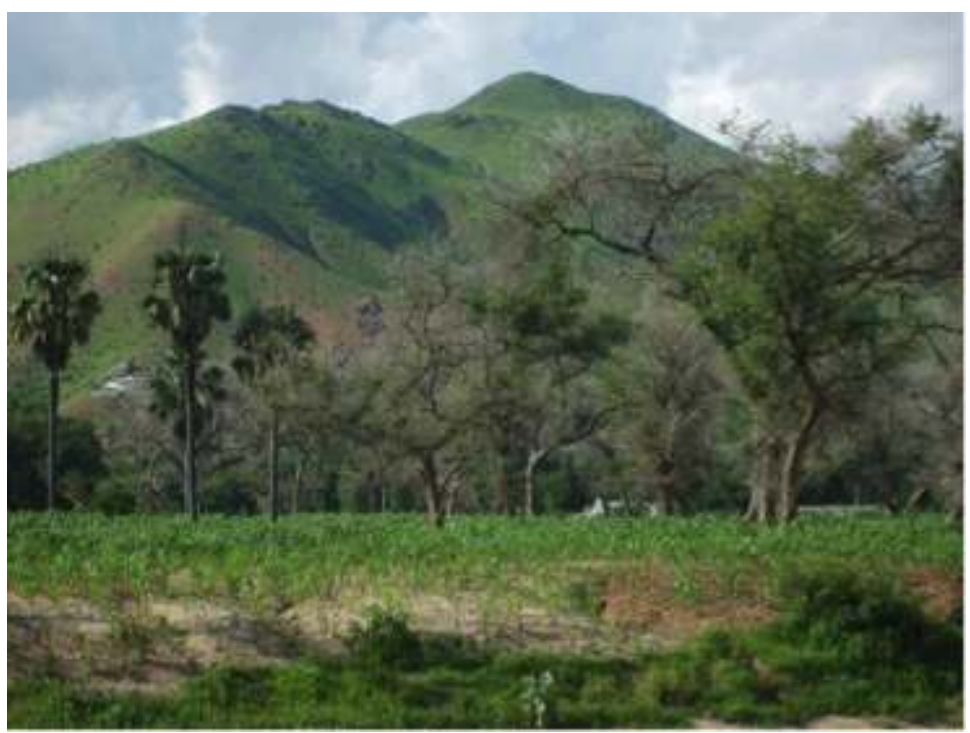

Figure 4. Parc à plusieurs essences (Tamarindus indica, Mangifera indica, Citrus limon, Vitellaria paradoxa, Sclerocarya birrea, etc.), Orodara (Burkina Faso), novembre 2013

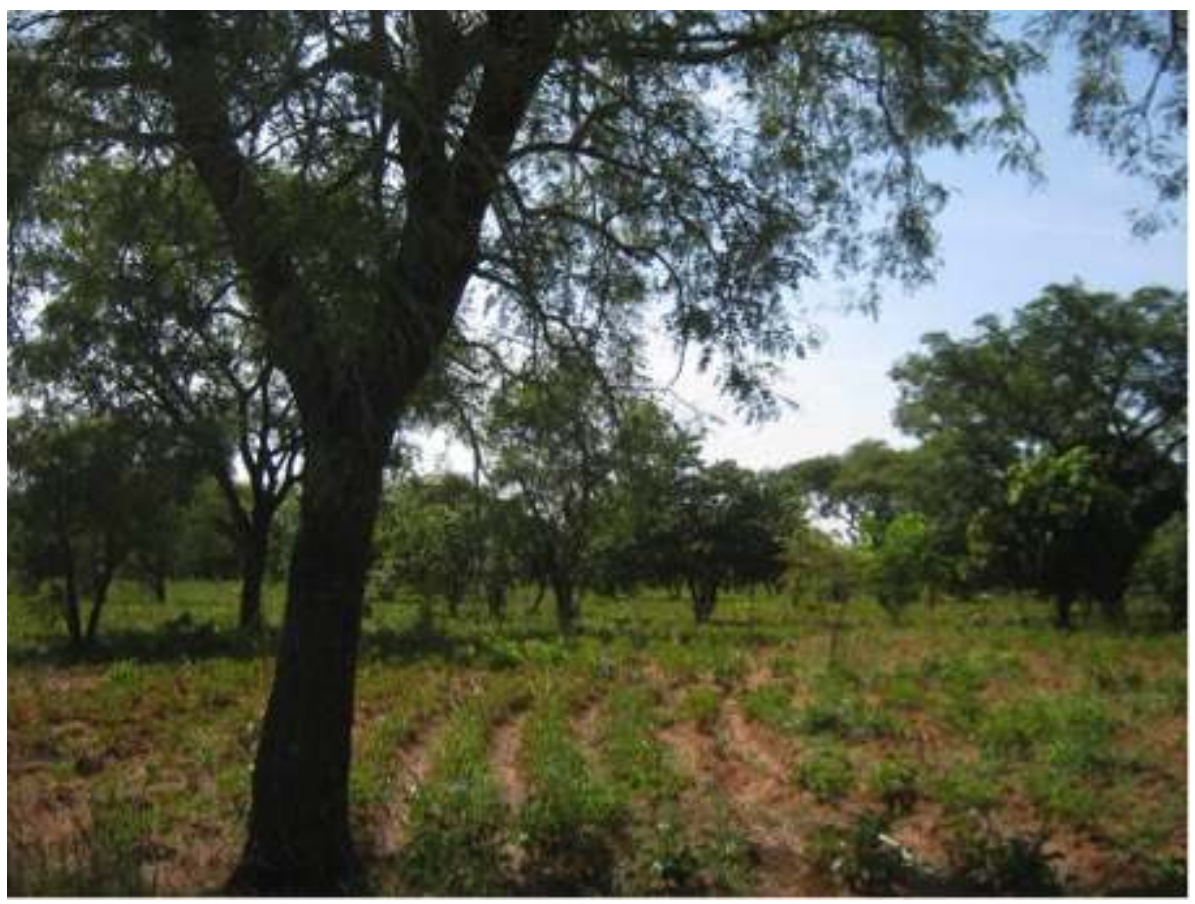

5 En effet, une des caractéristiques du parc est l'association couverture arborée/champ (des champs permanents ou semi-permanents). Jean-Paul Lahuec (1980) définit le parc comme «l'ensemble des espèces arborées figurant sur le terroir ». C'est un paysage construit par l'homme où l'arbre profite d'une gestion aussi méticuleuse que celle de la terre (Boffa, 1999; Huxley, 1999). Il s'agit d'un système d'utilisation des terres dans lequel les végétaux ligneux pérennes sont délibérément conservés en association avec les cultures et/ou l'élevage dans un arrangement spatial dispersé et où existent à la fois des interactions écologiques et économiques entre les ligneux et les autres composantes du système (Picouet et al., 2004). Les arbres ne sont pas plantés mais sont 
le fruit d'une sélection réalisée par l'homme parmi les essences en place, complétées éventuellement par des semis, plants ou boutures. Les parcs sont formés d'une ou plusieurs espèces, l'une dominante, les autres secondaires ou "accompagnatrices». Leur densité d'arbres et leur composition sont extrêmement variables dans le temps et dans l'espace. Aussi le parc devient un objet précieux pour comprendre les sociétés dans leurs dynamiques sociospatiales. Les fonctions des parcs (chaque parc en a une ou plusieurs) finement décrites (Seignobos, 2014), très fluctuantes dans le temps et différentes d'un terroir à l'autre, sont en fait un reflet des peuples/sociétés qui en sont à l'origine.

- Les parcs de famine : Ficus spp., Borassus aethiopum. Ce sont des essences sélectionnées en priorité lors de l'installation d'un groupe et donc lors de la construction d'un agrosystème. Avec le temps, ces parcs sont ensuite étoffés par d'autres essences et les espèces de famine régressent lentement dans le pourcentage des arbres du parc.

- Les parcs d'appoint alimentaire sont formés d'essences codominantes interchangeables. Ainsi, la trilogie Parkia biglobosa, Tamarindus indica, Vitellaria paradoxa peut faire place à celle de Parkia biglobosa, Adansonia digitata, Ceiba pentandra, etc. Certains peuvent offrir un meilleur équilibre que d'autres et répondre à toutes les demandes alimentaires en marge des céréales. Par exemple, Vitellaria paradoxa comble les besoins en matières grasses, Tamarindus indica est utilisé pour la préparation de boissons et de bouillies. C'est également le cas de certains arbres utilisés comme des brèdes ou des légumes sauce (différentes variétés de légumes composés essentiellement de feuilles à l'image des épinards dans les régions tempérées) : feuilles fraîches ou sèches de Adansonia digitata, graines et pulpes des gousses de Parkia biglobosa, etc. Les arbres les plus recherchés sont ceux qui fournissent à la fois légumes verts et condiments huileux.

- Les parcs oléifères. La recherche de matières grasses est un souci constant. Outre les « huiles vestimentaires» (les populations s'en enduisaient le corps) dominées par celle de Khaya senegalensis et les huiles médicinales, ce sont aussi les huiles alimentaires qui sont concoctées avec Balanites aegyptiaca, Vitellaria paradoxa, Lophira alata, etc. D'autres productions sont également présentes, sous forme de condiments huileux : graines de Ceiba pentandra, de Adansonia digita, de Celtis integrifolia, de Sclerocarya birrea, etc.

- Les parcs vignobles. La recherche de boisson correspond à des productions annexes dans le parc. C'est le cas par exemple d'arbres à tout faire comme Borassum aethiopum ou les différents ficus.

- Les parcs vestimentaires. Adansonia digitata y joue un rôle de choix car les cordelettes faites avec l'écorce sert à confectionner les jupes des femmes. Des ficus, en particulier Ficus thonningii et Ficus glumosa, fournissent également des étoffes. Ce type de parc a aujourd'hui totalement disparu, n'étant plus en phase avec les habitudes vestimentaires.

- Les parcs répondant aux besoins en bois, surtout pour la construction des cases. Ils sont surtout composés de Ziziphus spp., Anogeissus leiocarpus.

6 Le parc intervient principalement dans le cadre de communautés rurales stables, groupées et à partir de certaines densités de population. Les fortes densités, exerçant une pression importante sur les espaces cultivables, incitent à une meilleure gestion des terres et à une sélection précise de l'arbre, tant quantitative que qualitative. La densité d'arbres dans un parc est extrêmement variable allant de quelques pieds à l'hectare à plus de 150. La composition arborée d'un parc et sa densité vont découler en grande partie de la densité du peuplement humain et de la nature de l'habitat. La corrélation entre la situation démographique et la nature des parcs reste fondamentale. Un habitat groupé et stable va favoriser la sélection quantitative et qualitative des 
arbres et donc la construction de parcs. Un habitat dispersé et nomade va en revanche en limiter la présence.

7 L'évolution socioéconomique des populations provoque la modification des parcs dont elles s'occupent : superficie, composition, densité, etc. La structure du parc évolue donc dans le temps. Le parc est adapté et constamment modifié pour subvenir aux nouveaux besoins. Il peut également s'appauvrir et disparaitre si le nouveau mode de vie de la population l'impose. Les parcs en mutation présentent des formes d'emboîtement et de juxtaposition. La diffusion de certaines cultures, en particulier celles qui fournissent des matières grasses (arachide, sésame, oseille de Guinée) ou des aliments pour la sauce (gombo) ont entraîné la disparition, dans certains parcs au nord du Cameroun, de tous les arbres fournisseurs de matières grasses, de condiments, comme Vitellaria paradoxa, Sclerocarya birrea, Balanites aegyptiaca, Adansonia digitata, Ceiba pentandra, excepté lorsqu'ils sont utilisés pour d'autres services.

8 Au final, les espèces et les formes de boisement ne se ressemblent pas et chaque champ présente une particularité. Les différentes espèces sont souvent connues pour leurs vertus thérapeutiques, agronomiques, leur signification symbolique, etc. Un paysan disait: "Plus il y a d'arbres, plus la terre est bonne et plus il y a de pluie.» Les boisements dans les champs ne sont pourtant pas les mêmes. Chaque espèce, chaque arbre, a un rôle bien particulier (tableau 1).

Tableau 1. Les arbres présents dans les champs à Barani et leurs usages actuels

\begin{tabular}{|c|c|c|c|}
\hline $\begin{array}{l}\text { Nom } \\
\text { français }\end{array}$ & $\begin{array}{l}\text { Nom latin } \\
\text { (famille) }\end{array}$ & Caractéristiques & $\begin{array}{l}\text { Usages agroforestiers } \\
\text { actuels }\end{array}$ \\
\hline $\begin{array}{l}\text { Arbre blanc, } \\
\text { cad, } \\
\text { faidherbier }\end{array}$ & $\begin{array}{l}\text { Acacia albida } \\
\text { ou Faidherbia } \\
\text { albida }\end{array}$ & $\begin{array}{l}\text { Grand épineux de savane africaine, } \\
\text { phénologie inversée, racines profondes: } \\
\text { non concurrence avec les cultures en } \\
\text { place. }\end{array}$ & $\begin{array}{l}\text { Clôtures bois sec, haies } \\
\text { vives, ombrage, } \\
\text { fourrage }\end{array}$ \\
\hline- & Acacia hockii & $\begin{array}{l}\text { Petit arbre ou arbuste épineux à cime } \\
\text { étalée et ouverte, floraison en début de } \\
\text { saison sèche. }\end{array}$ & Fourrage \\
\hline $\begin{array}{l}\text { Gommier } \\
\text { rouge }\end{array}$ & Acacia nilotica & $\begin{array}{l}\text { Arbre épineux atteignant } 20 \text { mètres de } \\
\text { haut; espèce de sols lourds et mal } \\
\text { drainés ; graines transformées en colle. }\end{array}$ & $\begin{array}{l}\text { Clôtures bois sec, haies } \\
\text { vivres, fourrage }\end{array}$ \\
\hline $\begin{array}{l}\text { Dattier du } \\
\text { désert }\end{array}$ & $\begin{array}{l}\text { Balanites } \\
\text { aegyptiaca }\end{array}$ & $\begin{array}{l}\text { Petit arbre à feuilles persistantes vert } \\
\text { foncé; fruits comestibles. }\end{array}$ & $\begin{array}{lll}\text { Clôtures bois } & \text { sec, } \\
\text { fixation des } & \text { sols, } \\
\text { fourrage } & & \end{array}$ \\
\hline Baobab & $\begin{array}{l}\text { Adamsonia } \\
\text { digitata }\end{array}$ & $\begin{array}{l}\text { Très reconnaissable; jusqu'à } 25 \text { mètres de } \\
\text { hauteur; feuilles utilisées en cuisine et } \\
\text { graines consommées comme bonbon ou en } \\
\text { jus. }\end{array}$ & Usage alimentaire \\
\hline Caiilcédrat & $\begin{array}{l}\text { Khaya } \\
\text { senegalensis }\end{array}$ & Jusqu'à 30 à 35 mètres. & $\begin{array}{l}\text { Ombrage et usage } \\
\text { médicinal }\end{array}$ \\
\hline
\end{tabular}




\begin{tabular}{|c|c|c|c|}
\hline Citronnier & Citrus limon & $\begin{array}{l}\text { Petit arbre épineux à feuilles persistantes } \\
\text { de } 3 \text { à } 8 \text { mètres de haut. }\end{array}$ & Ombrage \\
\hline Eucalytpus & $\begin{array}{l}\text { Eucaluptus } \\
\text { camaldulensis }\end{array}$ & $\begin{array}{l}\text { Arbre à feuilles persistantes, atteignant } 12 \\
\text { à } 20 \text { mètres. }\end{array}$ & $\begin{array}{l}\text { Bornage, clôtures bois } \\
\text { sec, haies vives, } \\
\text { ombrage, ornemental, } \\
\text { reboisement, fourrage }\end{array}$ \\
\hline Jujubier & $\begin{array}{l}\text { Ziziphus } \\
\text { mauritiana }\end{array}$ & $\begin{array}{l}\text { Arbuste buissonnant et épineux; fruits } \\
\text { comestibles: les jujubes. }\end{array}$ & $\begin{array}{l}\text { Brise-vent, clôtures } \\
\text { bois sec, haies vives, } \\
\text { ombrage }\end{array}$ \\
\hline $\begin{array}{l}\text { Kapokier } \\
\text { rouge }\end{array}$ & $\begin{array}{l}\text { Bombax } \\
\text { costatum }\end{array}$ & Environ 20 mètres, fût droit, fruits utiles. & Usage alimentaire \\
\hline Karité & $\begin{array}{l}\text { Vitellaria } \\
\text { paradoxa }\end{array}$ & $\begin{array}{l}\text { Arbre trapu à rameaux épais, pulpe du } \\
\text { fruit comestible, amende riche en graisse } \\
\text { (beurre de karité) }\end{array}$ & $\begin{array}{l}\text { Usage alimentaire et } \\
\text { cosmétique }\end{array}$ \\
\hline Kinkeliba & $\begin{array}{l}\text { Combretum } \\
\text { micranthum }\end{array}$ & $\begin{array}{l}\text { Arbuste touffu et buissonnant; } 4 \text { à } 5 \\
\text { mètres, pyrorésistant. }\end{array}$ & $\begin{array}{l}\text { Usage alimentaire et } \\
\text { médicinal }\end{array}$ \\
\hline Neem & $\begin{array}{l}\text { Azadirachta } \\
\text { indica }\end{array}$ & $\begin{array}{l}\text { Provenance : Indes ou Iran; diffusion } \\
\text { rapide; agent de diffusion : chauve-souris. }\end{array}$ & $\begin{array}{l}\text { Brise-vent, engrais, } \\
\text { ombrage fertilisation, } \\
\text { ornemental, } \\
\text { restauration des sols }\end{array}$ \\
\hline Néré & Parkia biglobosa & $\begin{array}{l}\text { De } 10 \text { à } 15 \text { mètres de haut, très utilisé } \\
\text { (feuilles consommées en poudre, fleurs } \\
\text { sucées, fruits transformés en jus, graines } \\
\text { fermentées pour condiment, etc.; } \\
\text { traditionnellement protégé, fertilise les } \\
\text { sols grâce à ses feuilles caduques. }\end{array}$ & Usage alimentaire \\
\hline $\begin{array}{l}\text { Palmier } \\
\text { doum }\end{array}$ & $\begin{array}{l}\text { Hyphaene } \\
\text { thebaica }\end{array}$ & $\begin{array}{l}\text { Palier de } 12 \text { à } 15 \text { mètres de haut, amandes } \\
\text { mangées crues ou cuites. }\end{array}$ & Usage alimentaire \\
\hline $\begin{array}{l}\text { Palmier } \\
\text { rônier }\end{array}$ & $\begin{array}{l}\text { Borassus } \\
\text { aethiopum }\end{array}$ & $\begin{array}{l}\text { Palmier atteignant } 30 \text { mètres de haut; } \\
\text { bas-fonds inondés temporairement. }\end{array}$ & Usage alimentaire \\
\hline Marula & $\begin{array}{l}\text { Sclerocarya } \\
\text { birrea }\end{array}$ & $\begin{array}{l}\text { Arbre moyen; développement en } \\
\text { couronne ; fruits fortement utilisés. }\end{array}$ & Haies vives \\
\hline Tamarin & $\begin{array}{l}\text { Tamarindus } \\
\text { indica }\end{array}$ & $\begin{array}{l}10 \text { à } 20 \text { mètres; croissance lente ; feuilles } \\
\text { persistantes ; pulpe utilisée pour jus. }\end{array}$ & Ombrage \\
\hline
\end{tabular}

9 Les arbres ne sont pourtant pas tous les bienvenus dans les champs. Les croyances locales peuvent en être l'une des explications. Sclerocarya birrea par exemple est accusé d'empêcher le bon développement des cultures à ses pieds, et entraîne une baisse des rendements. Hyphaene thebaica serait, selon des paysans, assez néfastes pour les cultures. Certains épineux acidifient le sol et donc altèrent les cultures. Bombax 
costatum, quant à lui, est parfois considéré comme ayant une relation saine avec les cultures alors que d'autres diront qu'il augmente la présence d'une herbe appelée localement potasse (Kata en fulfulde ${ }^{2}$ et Seguin en Dioula) dont tous les paysans souhaitent se débarrasser. Cette herbe ralentit très fortement la croissance du mil, mais entre dans la composition de la potasse, utilisée en cuisine. Les arbres sont néanmoins conservés du fait de leur poids symbolique, de l'interdiction d'abattage par les services municipaux ou tout simplement du fait du manque d'outillage adapté pour l'abattage. Au final, le parc est une sécrétion de l'ethnie, c'est-à-dire une production non planifiée issue des habitudes alimentaires et des modes de vie comme peuvent également l'être l'organisation du terroir, l'éventail des cultures ou l'architecture (figure 5).

Figure 5. Les arbres (Tamarindus indica, Faidherbia albida, Azadichrata indica, Ziziphus mauritiana, etc.) dans le terroir villageois de la région de Mokolo (Cameroun), mars 1998

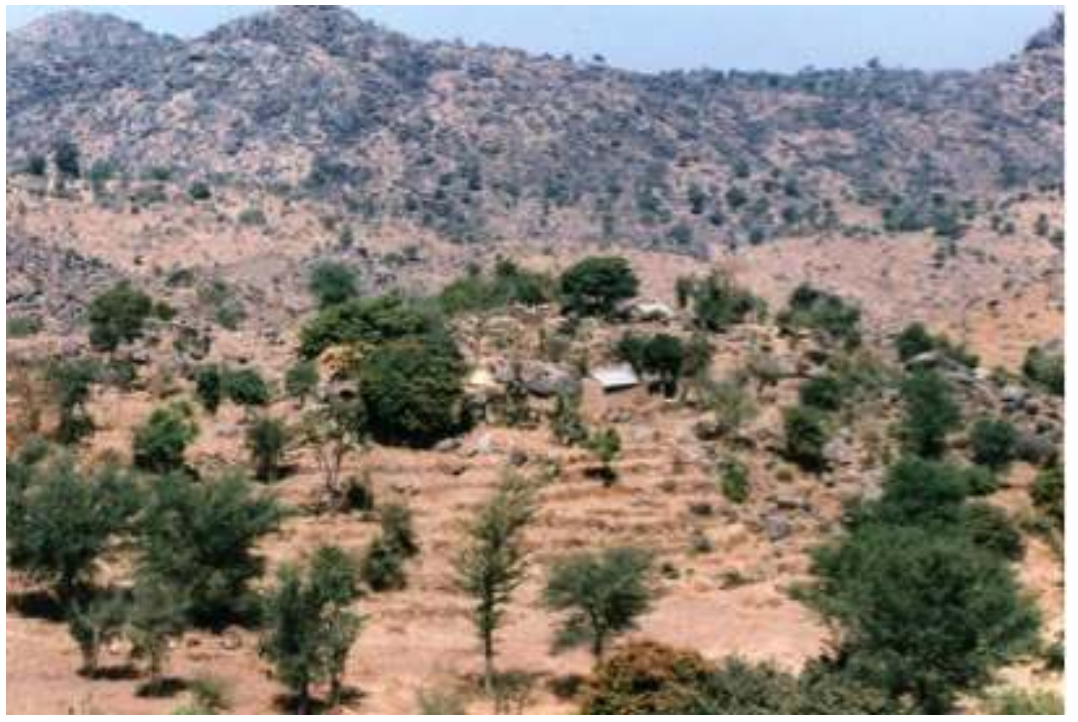

10 Le paysage qui en résulte sera un espace plus ou moins boisé dont la signification échappera totalement à l'œil non averti. En absence des cultures en saison sèche, il peut être facilement confondu à la savane qui pourtant revêt une autre réalité.

\section{L'arbre des stratégies de survie}

11 À l'extérieur des parcelles cultivées, les arbres ne sont plus directement sélectionnés par les hommes. Ce sont des savanes plus ou moins riches en arbres (Ballouche, 2016), que la population locale appelle «forêts » (Yengué et Cochonneau, 2013) (figure 6). En matière de densité et de composition floristique, elles peuvent ressembler à s'y méprendre aux parcs. La différence fondamentale entre les deux est leur place dans les habitudes de vie. «La forêt » est parcourue par les villageois à la recherche de produits divers, perches pour la construction, miel, chasse, bois de feu, etc. En saison pluvieuse, période des cultures, c'est ici que le bétail passe le plus clair de son temps. Dans le nord-ouest du Burkina Faso, ces «forêts » se composent aussi de jachères, largement décrites par de nombreux auteurs (Nkamleu, 1999; Floret, 2001 Guengant et al., 2006 ; Devineau et Serpantié, 1991). 
Figure 6. Savane arbustive (Balanites aegyptiaca) à Barani (Burkina Faso), février 2012

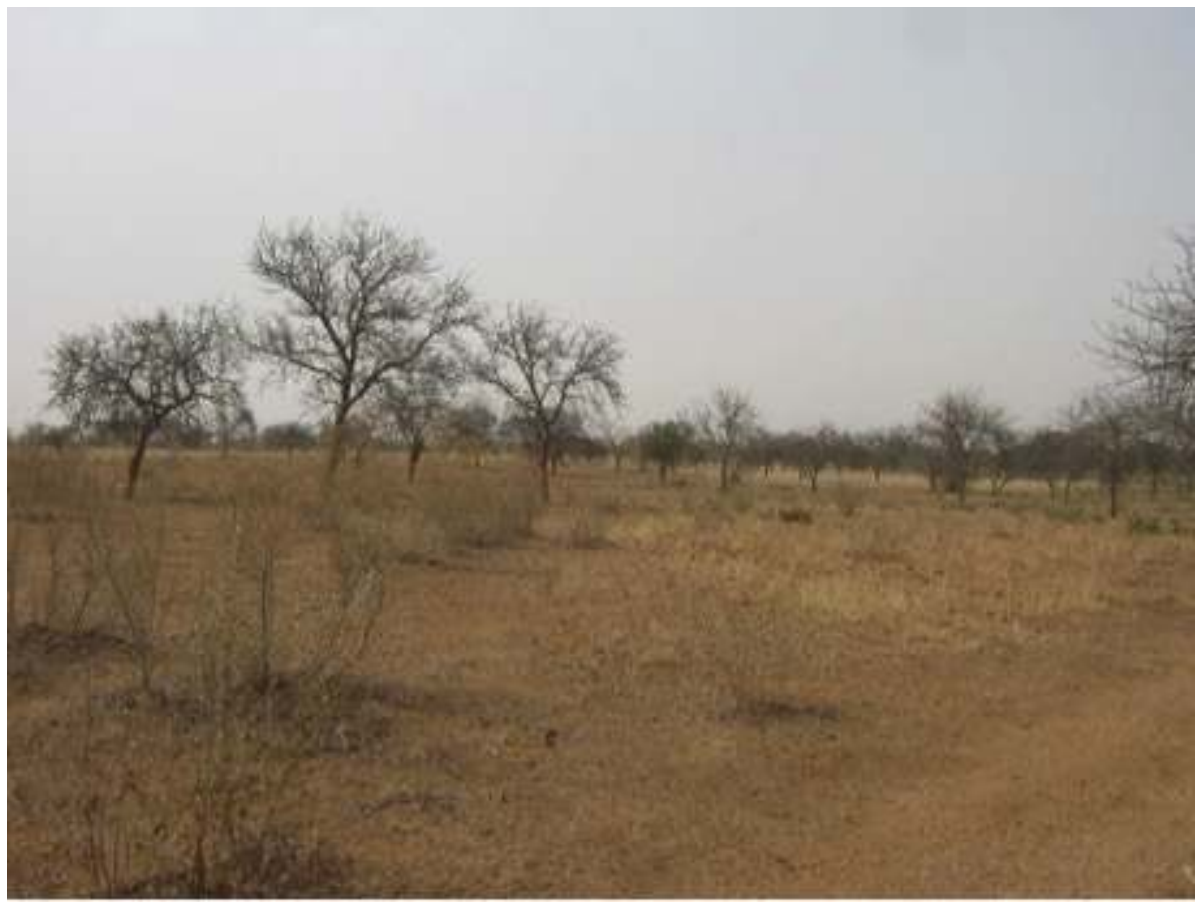

Après plusieurs années consécutives de culture, la terre se fatigue et «trahit », comme disent les paysans de Barani. Les rendements ne sont plus au rendez-vous. Il est alors temps de la laisser au repos. Malgré tout, la jachère n'est pas unique et chaque paysan a ses propres méthodes et fréquences. À Barani, le repos peut durer jusqu'à 50 ans, sous des formes nombreuses et subtiles, en lien avec la localisation de la parcelle dans le terroir, la complémentarité entre les terres d'un même agriculteur, l'environnement du champ, des cultures pratiquées, la composition de la famille, les conditions météorologiques, etc. Ces jachères peuvent être organisées en plusieurs catégories (Yengué, 2015 ; Yengué et Cochonneau, 2013) :

- Les agriculteurs disposant de peu de parcelles ne pratiquent pas ou peu le repos du sol par manque de terre, ce qui rend la jachère impossible.

- Quand le matériel se fait rare (charrues non réparées pendant la morte-saison, animaux de traction défaillants, semences rares) ou que les moyens humains viennent à manquer (maladie, exode rural), le travail des champs devient difficile. Alors, des stratégies combinant la quantité d'eau tombée, la qualité du sol, les forces de travail se mettent en place pour estimer la bonne surface à exploiter. Les agriculteurs ne peuvent pas se permettre d'engager du temps, des semences et parfois de l'argent pour des cultures qui ont de fortes chances de ne pas aboutir. Ils en profitent donc pour laisser des parcelles au repos. C'est la jachère contrainte.

- Dans d'autres cas encore, la jachère est maintenue pour les produits qu'elle procure: le fourrage pour les animaux, les fruits et baies sauvages pour améliorer le quotidien, le bois pour la construction ou pour la confection des manches d'outil, les feuilles et autres écorces pour la pharmacopée, etc. C'est bien la fourniture de ces produits qui est le motif premier du maintien de la jachère, bien avant la régénération du sol. C'est ce que nous qualifions la jachère utile.

- Certaines parties du terroir sont totalement délaissées. La culture ne pourrait à nouveau y être pratiquée qu'au prix de profonds changements dans les pratiques. C'est notamment le 
cas des abords des parcours de transhumance, où la terre est tassée en raison du passage répété des animaux. Ces parties du terroir sont abandonnées faute de pouvoir être exploitées. Elles sont donc laissées à la dynamique spontanée de la végétation. Ce sont des jachères d'abandon.

- Enfin, et ce n'est pas le cas le plus courant à Barani, il existe la jachère choisie ou encore la jachère pour la régénération du sol, bien décrite par Floret (2001). À Barani, la durée de cette jachère ne peut être très longue à cause, entre autres, de la pression sociale. Le long repos attire la convoitise des voisins. Le propriétaire s'astreint donc à une culture permanente ou à réduire la durée de sa jachère pour ne pas être obligé de prêter ses terres. En effet, il est fréquent d'être sollicité par les voisins « lorsqu'on peut se payer le luxe de laisser ses terres sans cultures ».

Sur le plan spatial, les jachères les plus anciennes (plus de 10 ans) et les plus grandes en superficie vont se retrouver les plus éloignées du village. Il s'agit le plus souvent des jachères utiles. Au fur et à mesure qu'on se rapproche du village, les jachères seront de courte durée (jachères contraintes). Les champs les plus proches des habitations (les champs de cases), quant à eux, seront exploités sans repos. Non seulement ce sont les plus faciles à cultiver puisque la distance à parcourir est courte mais en plus ils bénéficient souvent d'un traitement «de faveur » comme un amendement supérieur par exemple. Seules les jachères d'abandon vont se retrouver sur tout le terroir.

La mise en repos permet la reprise de la dynamique naturelle de la végétation. Le résultat est une formation végétale qui peut, plus ou moins vite, avoir le profil d'une savane jamais cultivée. Ces «forêts » sont parcourues et (sur) exploitées pour répondre à des besoins toujours plus grands. La savane jamais cultivée est de plus en plus grignotée pour l'extension des champs. La demande foncière toujours plus forte entraîne une réduction du temps consacré au repos des sols. Ces «forêts » tendent à mieux se maintenir sur les terres incultes et sur des espaces non ou difficilement accessibles (zones protégées, zone d'insécurité, zones éloignées des espaces habités) comme c'est le cas au nord du Cameroun (Yengué, 2002). La distribution spatiale et la composition des savanes sont finalement la résultante de l'agriculture et de la sédentarisation des peuples qui se concentrent dorénavant dans les villes et les gros villages. S'y trament alors des jeux de pouvoir et de domination complexes.

\section{L'arbre du pouvoir et de la domination}

15 La différence d'aspect entre les agglomérations et les lieux non habités est très marquante dans le Nord du Cameroun comme au Burkina Faso (figure 7, 8 et 9). Les cités s'étendent sous de grands arbres, leur donnant l'aspect d'oasis en plein désert, surtout en saison sèche. Ici, presque toutes les rues sont bordées d'arbres. Seules des espèces sempervirentes sont plantées. Ces espèces gardent leurs feuilles toute l'année et permettent ainsi d'avoir de l'ombre pendant la rude saison sèche, où le thermomètre affiche souvent plus de $50^{\circ} \mathrm{C}$. 
Figure 7. Maroua (Cameroun), sous son couvert de Azadirachta indica, au bord du Mayo-Tsanaga, la rivière (asséchée à cette période de l'année) qui traverse la ville, mars 2013

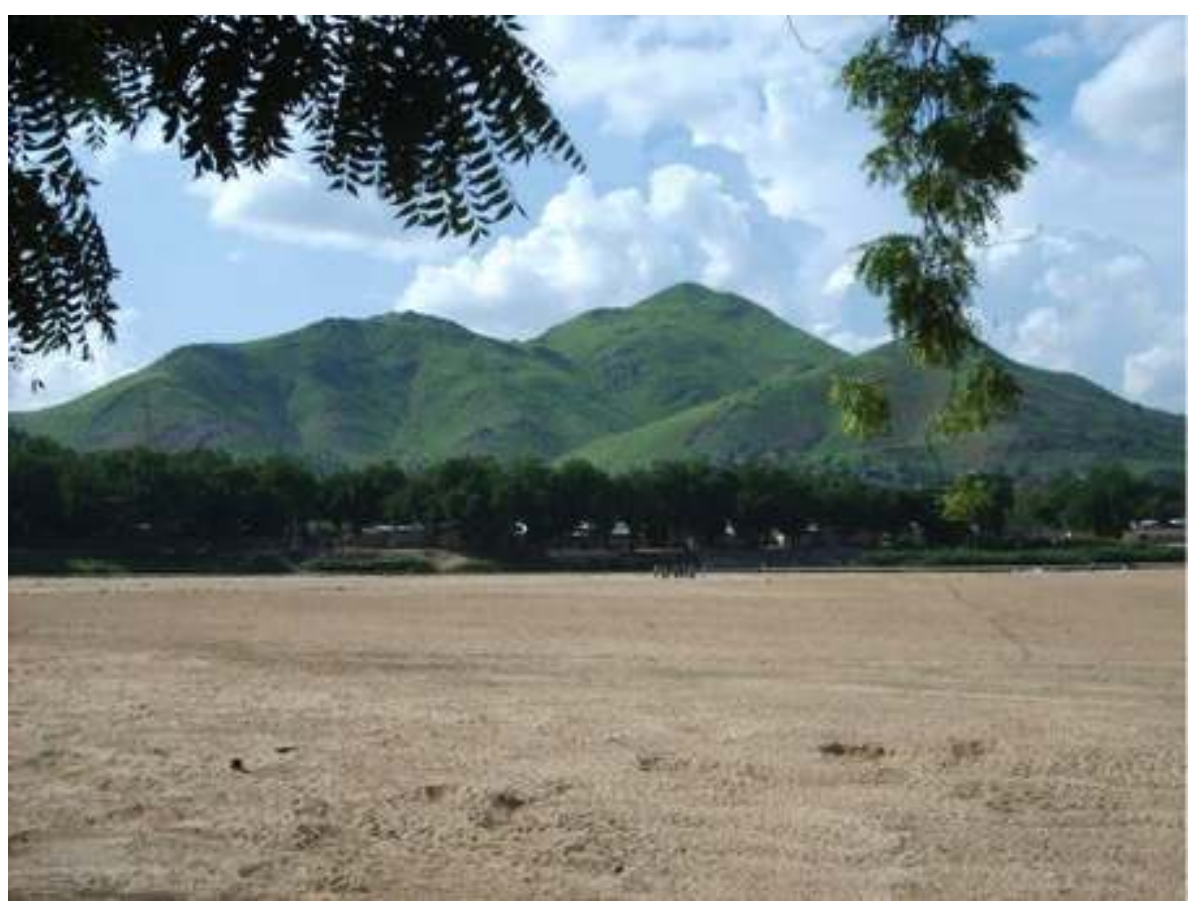

Figure 8. Activités informelles sous des Kaya senegalensis dans une rue de Bobo-Dioulasso (Burkina Faso), février 2013

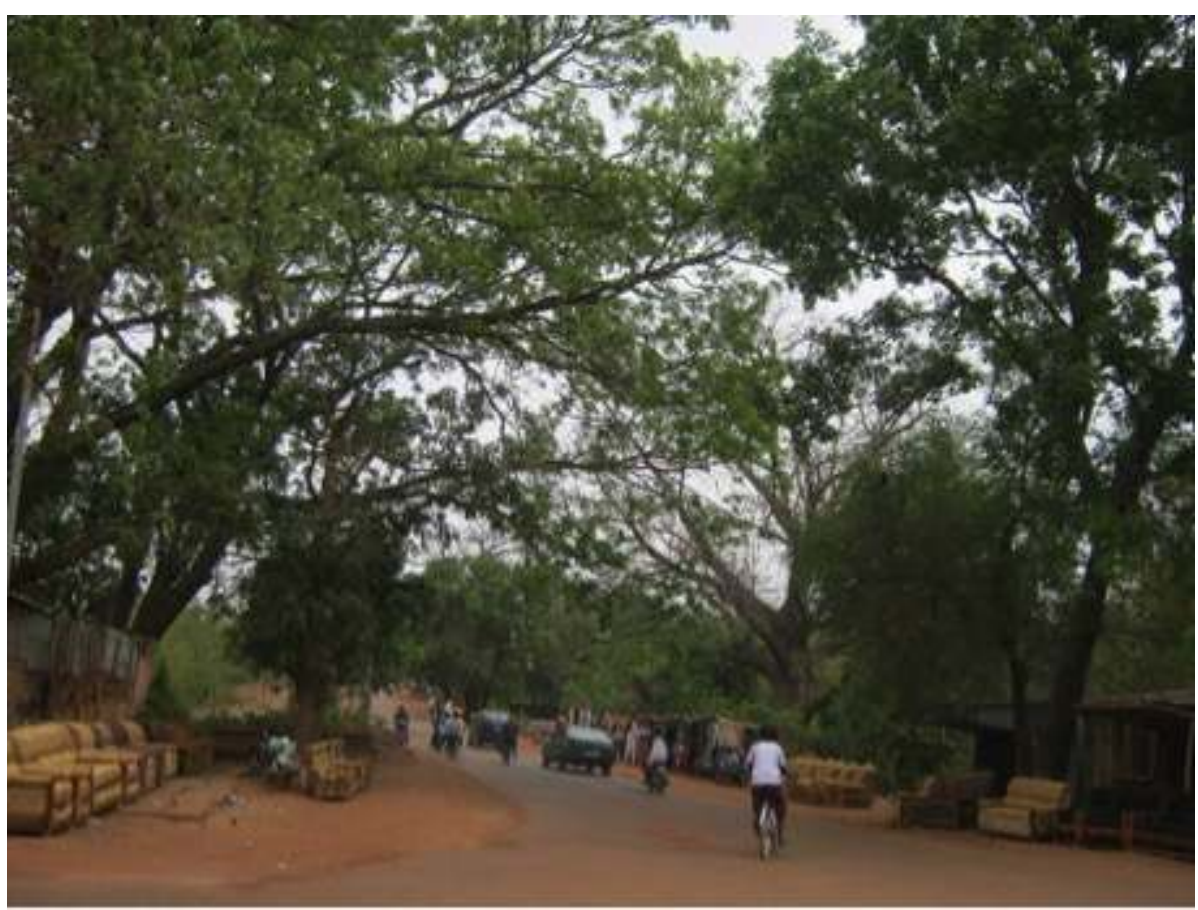


Figure 9. Les arbres dans un bas-fond de Yaoundé (Cameroun), décembre 2015

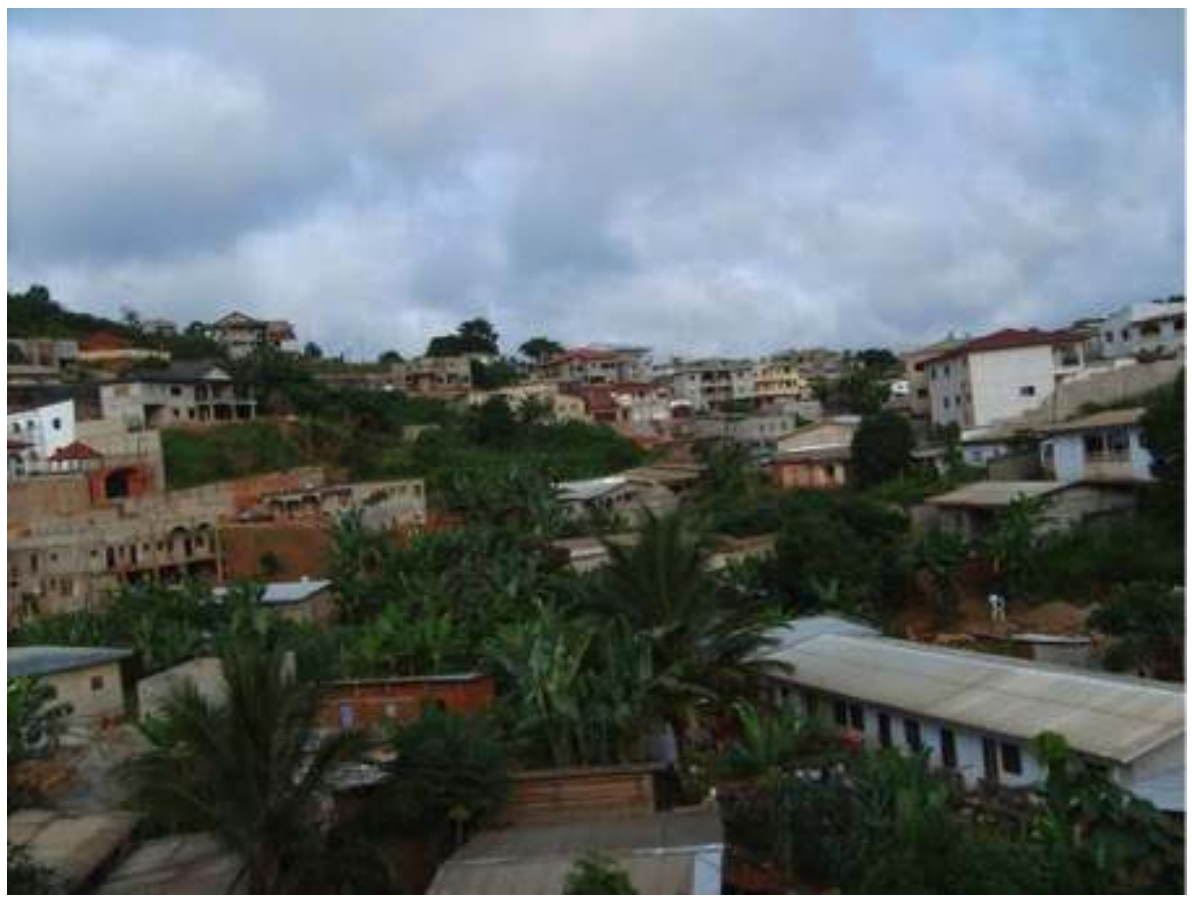

Toutes les activités dites informelles se développent à l'ombre de ces arbres. Les rues les plus ombragées sont les plus animées et regorgent d'activités multiples. À l'inverse, les voies les plus exposées aux rayons du soleil sont les plus délaissées. Les commerçants refusent d'ailleurs de se faire des abris, très souvent en tôle ou avec des matériaux de récupération, car l'abri a de fortes chances d'être balayé dès les premières rafales de l'harmattan. Ils préfèrent proposer leurs services ou leurs articles à l'ombre d'un arbre couvert de feuilles. Puisqu'il ne pleut que très rarement, aucun problème ne se pose, bien au contraire. Les espèces les plus rencontrées sont des ficus, palmiers doum, dattiers, acacias, flamboyants, Khaya senegalensis, etc. La palme revient à Azadirachta indica omniprésent dans pratiquement toutes les villes et les villages du Burkina Faso et du Nord du Cameroun. Pratiquement toutes les implantations humaines pérennes sont marquées par la présence de Azadirachta indica. Dans la partie montagneuse du Nord du Cameroun (les monts Mandara), la pénétration de Azadirachta indica n'est pas de même ampleur, la montagne étant longtemps restée repliée sur ellemême et donc s'étant très peu ouverte aux nouvelles pratiques. Seuls les villes et les gros villages sont complantés de Azadirachta indica. Mais de plus en plus, cette espèce étend ses ramifications même dans les endroits les plus reculés de la montagne. En zone humide (Yaoundé et Bafoussam), on trouvera des eucalyptus, différents fruitiers (Persea americana, Citrus limon, Mangifera indica) et des Elaeis guineensis. Depuis une vingtaine d'années, d'autres essences se développent pour leur forme (Ravenala madagascariensis) ou leur floraison odorante (Cananga odorata).

Le caractère fortement boisé des lieux d'habitation en Afrique sèche est un phénomène relativement nouveau. Dans les pratiques ancestrales et traditionnelles, l'arbre est généralement rejeté hors de la cité (Yengué et Callot, 2002). L’arbre est accusé de faire pourrir le chaume des toits qu'il recouvre, d'être incompatible avec les cases de terre, car il empêche le soleil de les sécher rapidement après la pluie, de servir souvent de perchoirs à des colonies d'oiseaux dont les déjections constituent une grande gêne, 
d'abriter les animaux de mauvais augure tels que les hiboux et autres oiseaux nocturnes, etc. Dans certaines ethnies, l'arbre est considéré comme le trait d'union entre les dieux représentés par les morts (dans les pratiques animistes, les dieux sont dans le sol, là où sont enterrés les défunts) et les vivants. Il a donc un caractère mystique entre ses racines qui conversent avec les dieux et son houppier qui pacte avec les vivants. Au nord du Cameroun par exemple, planter un arbre, d'autant plus dans les lieux habités, est un acte sacré, prérogative de quelques initiés (chefs, forgerons et autres personnes habilitées). Aussi, la fracture entre les lieux habités et le reste du paysage est aussi nette qu'aujourd'hui, mais en négatif, les agglomérations étant dénudées ou au mieux ponctuées d'un réseau très lâche d'arbres. En effet, l'arbre n'est pas totalement absent de la cité. Quelques espèces utiles peuvent être tolérées, notamment pour leur intérêt médicinal, alimentaire, sacré, etc. C'est le cas de certains ficus (Ficus spp.) qui ombragent non pas les concessions privées, mais les différentes places des bourgs. Ils sont sacralisés par leur implantation à proximité de la maison du chef. Ce sont des essences à diffusion facile par bouturage. Le vainqueur qui occupe un village fait de temps à autre des prélèvements afin de les bouturer chez lui pour se rappeler l'exploit. Parfois, c'est une variété tout entière qui est vénérée. C'est le cas de Ficus platyphylla, indifféremment à l'intérieur et hors des cités du Nord du Cameroun.

Le rapport des populations locales à ces arbres de ville peut dérouter. Utiles à l'activité économique et à la vie tout simplement, ils ne sont pourtant pas épargnés par les assauts de la hache. Il est fréquent de voir des espèces mutilées et d'assister à des coupes sauvages de la part des riverains. Le bois sert à alimenter le feu dans les cuisines, les feuilles sont utilisées pour la pharmacopée, les fruits pour la consommation, la sève pour des pratiques chamaniques, etc. L'explication de ce comportement ne réside pas seulement dans les difficiles conditions économiques, mais surtout dans l'histoire de ces formations boisées et leur rapport à la domination, au pouvoir.

19 C'est l'administration coloniale qui fit entrer l'arbre dans la ville dès le début du $\mathrm{xx}^{\mathrm{e}}$ siècle. Pour elle, l'agrément d'une ville se mesure à sa verdure conformément aux différents courants de pensée alors présents en Europe (Mehdi et al., 2012 ; Agulhon et Duby, 1998) :

- le courant hygiéniste qui se penche sur les conditions des laissés pour compte de la révolution industrielle $\mathrm{du}{ }_{\text {xviii }}{ }^{\mathrm{e}}$ siècle, i.e la classe ouvrière aux conditions de vie très difficiles. La végétation en ville est utilisée ici pour assainir la ville et la rendre plus vivable pour les plus pauvres ;

- le néoclassicisme qui prône le gigantisme dans le dessin de la ville et l'omniprésence du végétal pour souligner le caractère imposant de l'urbanisme.

Aussi, lors des épopées coloniales de la fin du XIX ${ }^{e}$ siècle, planter des arbres, c'est avoir d'une certaine façon prise sur la ville. C'est le moyen le plus immédiatement perceptible de la transformer. Pour les administrateurs qui n'effectuaient généralement que de courts séjours, les arbres plantés représentaient souvent la seule marque tangible de leur passage. Cela se traduit dans les villes par l'omniprésence de l'arbre et par la non-continuité des essences, chaque administrateur ayant eu sa préférence. Aussi, la répartition des essences est aléatoire dans les tissus urbains, sans localisation préférentielle de telle ou telle espèce dans les différents quartiers. Il est d'ailleurs fréquent d'avoir plusieurs espèces différentes sur une même rue. 
Cette arrivée de l'arbre en milieu urbain s'est d'abord faite dans les grandes villes, lieux de résidence des gouverneurs. En quelques années, le phénomène s'est répandu même dans les bourgades les plus petites. Pour les chefs locaux, c'était signe de modernité mais aussi signe de dépossession de leur pouvoir et donc d'allégeance à l'autorité colonisatrice. Pour autant, l'usage de l'arbre s'est démocratisé dans les cités, on en retrouve aujourd'hui dans tous les lieux d'habitation et même près des cases les plus isolées.

Certains administrateurs ont préféré introduire des espèces venant d'ailleurs à l'image de Azadirachta indica. Cette essence, omniprésente dans pratiquement tous les villages et les villes, a été introduite par le biais des colonies anglaises et en particulier le Nigeria (Seignobos, 1981). Elle toucha le Nord-Cameroun en 1947. C'est l'arbre de la facilité : il pousse rapidement, dans des conditions diverses de sol et aussi bien en milieu soudanien que sahélien. Implanté d'abord sur les marchés, puis le long des rues, il entra ensuite dans les concessions. Il peut se diffuser rapidement avec comme agent propagateur la chauve-souris qui raffole de ses fruits. Azadirachta indica est devenu peu à peu l'arbre de la ville et l'arbre de l'administration. En planter dans les villages, le long des axes principaux, sur les marchés sera un signe de l'emprise de la puissance coloniale et ensuite des États indépendants sur leur territoire. Aussi, pratiquement toutes les implantations humaines pérennes sont marquées par la présence de Azadirachta indica.

3 D'autres préféraient des arbres locaux d'allure imposante comme Kaya senegalensis. Contrairement à Azadirachta indica, cette essence est connue en Afrique sèche depuis des siècles. Son usage est donc complètement entré dans les mœurs. L'écorce de cet arbre est utilisée pour relever le goût de la bière de mil, et surtout pour la pharmacopée : traitement contre la jaunisse, les piqûres de scorpions, les maladies de la peau, les maux de tête et de ventre. Les racines secondaires, très près de la surface pour assurer l'équilibre de l'arbre, sont également recherchées. Elles servent de remède contre la stérilité, les maladies mentales, la syphilis et la lèpre. Les fruits rentrent dans la préparation d'une huile qu'utilisaient les femmes, autrefois habillées juste d'un cache-sexe, pour leur beauté corporelle.

Du fait de ces multiples usages, Kaya senegalensis est l'un des arbres qui tient une place privilégiée dans les sociétés animistes. La preuve en est que c'est l'une des rares essences à avoir un droit complètement différent de celui du sol. Le propriétaire de l'arbre n'est pas forcément celui qui détient la terre: un champ peut se vendre, peut s'échanger, mais Kaya senegalensis qui s'y trouve sera toujours la possession du premier propriétaire de la terre et de sa descendance. L'exploitation de l'arbre qui va de l'élagage au ramassage des fruits tombés lui reviendra toujours. Cet usage, généralisé avant la colonisation européenne, est de nos jours dépassé. Il reste quand même à l'origine de nombreux conflits dans certains villages du Nord du Cameroun. C'est la colonisation française qui va le faire entrer dans la ville comme arbre d'ornement (figure 10). 
Figure 10. Kaya senegalensis, érigé en monument de pouvoir, juste devant l'hôtel de ville de BoboDioulasso (Burkina Faso), février 2013

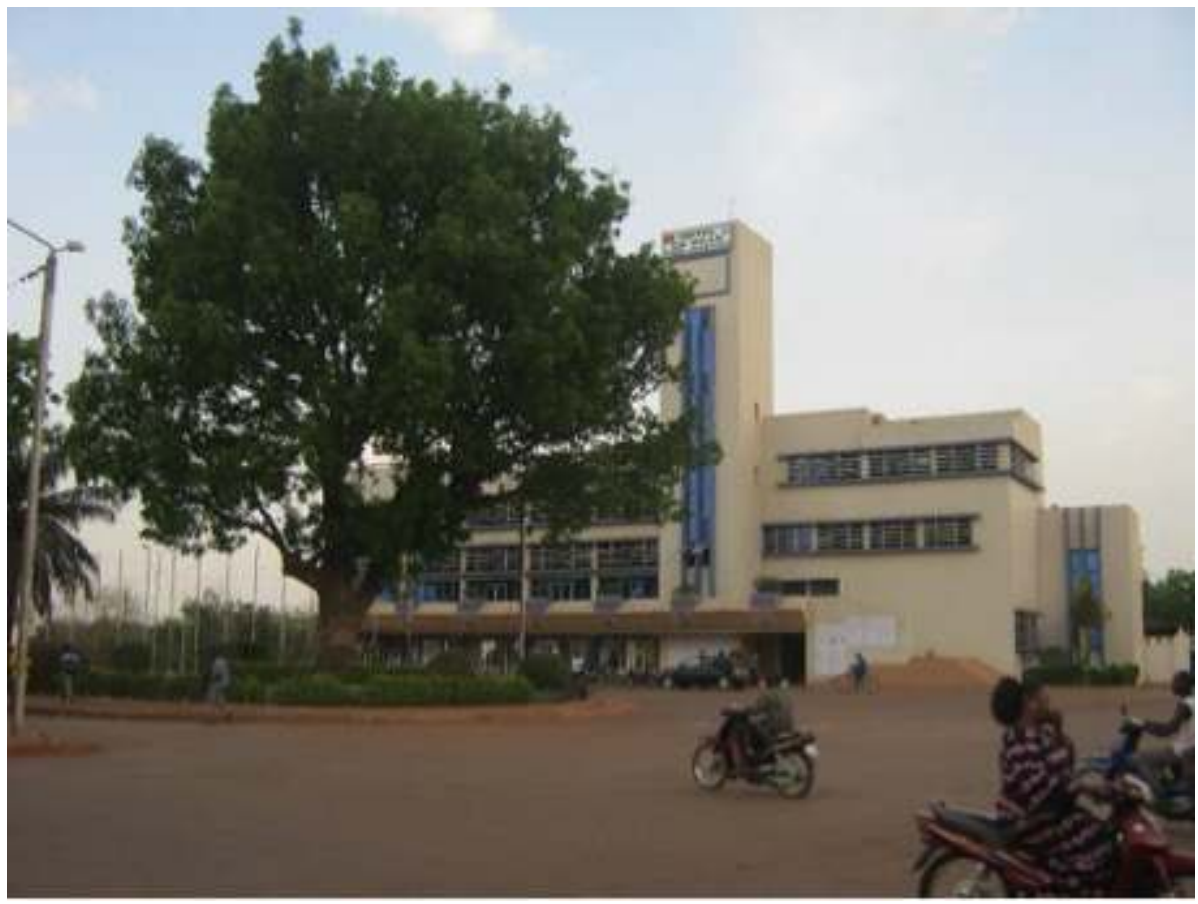

Seules quelques villes vont avoir de belles ruelles rectilignes bordées de Kaya senegalensis (Maroua au Cameroun, Ouagadougou et Bobo-Dioulasso au Burkina faso) L'utilisation de Kaya senegalensis comme arbre ornemental dans les villes sera très restreinte. Cet arbre ne se plante pas aisément et sa croissance est très lente, ce qui compromet son avenir dans les villes d'Afrique sèche.

Après la décolonisation dans les années 1960, ce sont les gouvernements et par la suite les services municipaux à qui reviennent dorénavant la plantation et l'entretien des arbres urbains. Les populations n'éprouvent pas d'attachement particulier à ces arbres. Ce sont les arbres du blanc (le colonisateur), du gouvernement, de la municipalité et ils sont donc considérés comme les arbres de l'autorité. À Yaoundé, cette place de l'arbre dans la conscience collective est utilisée par la communauté urbaine. Les bas-fonds marécageux, propriété de la collectivité selon le droit camerounais, sont systématiquement plantés en eucalyptus pour montrer la présence de la puissance publique et interdire, ou du moins limiter les bidonvilles.

Dans les sociétés précoloniales, la présence des arbres, aussi bien dans les champs que dans les lieux habités, ne s'explique que parce qu'ils ont une place dans les processus de vie et de survie. L'arbre-ornement est un legs de la colonisation européenne et sa diffusion ne s'est pas toujours faite pacifiquement. La mise en place du pouvoir colonial a engendré de fortes résistances locales violemment réprimandées. Aujourd'hui, la vision utilitariste de l'arbre perdure encore dans les villes et les villages étudiés. La population y voit bien plus qu'un élément du bien-être urbain, mais aussi une ressource qui viendrait améliorer le quotidien en cas de nécessité. C'est ce que nous avons étudié à Maroua, au nord du Cameroun (Yengué, 2006). Les spécimens de Kaya senegalensis plantés en alignement dans la ville ont été littéralement décimés (consommés) par les populations à la recherche de produits de pharmacopée traditionnels et de bois de chauffage à la suite de la crise économique du début des années 1990. La seule solution 
efficace pour enrayer cette destruction a été, pour la municipalité, de planter des essences étrangères, comme Azadirachta indica, sans fonction principale dans les usages locaux.

Introduit au Cameroun par l'administration coloniale française via le Nigéria, colonie britannique (Yengué, 2001), Azadirachta indica est originaire de l'Inde (Maydell, 1990). Il pousse là-bas depuis plusieurs siècles, et est couramment utilisé par les populations locales. Dans les colonies anciennes britanniques comme le Nigeria, il fut introduit il y a environ cent cinquante ans. Il a fallu une centaine d'années pour que les populations locales se l'approprient et l'exploitent comme une essence autochtone. Mais son utilisation est restée beaucoup plus restreinte qu'en Inde. Dans l'extrême nord du Nigeria, son bois est préféré aux autres espèces pour la production d'énergie. Toujours dans cette région, Azadirachta indica est planté en association avec les cultures en vue de fertiliser et d'améliorer la teneur en $\mathrm{pH}$ du sol. De plus, sous ces arbres, le sol est beaucoup plus humide pendant la saison sèche. Ainsi, il est de plus en plus utilisé en agroforesterie, dans les systèmes de rotation de culture avec une période de reboisement (Maydell, 1990)

Dans la région de Maroua, Azadirachta indica ne marque le paysage que depuis quelques années, à peine soixante ans. Son usage n'est donc pas encore entré dans les habitudes des populations locales. Ses feuilles sont parfois utilisées comme insecticide. De plus, pouvant se consumer même quand il vient d'être coupé, il constitue une réserve de bois de feu en cas de nécessité. Mais son exploitation est encore marginale, la fumée issue de sa combustion étant très abondante.

L'arbre, symbole de la domination, peut aussi être un objet de pacification et d'entente entre les peuples.

\section{L'arbre de la pacification}

Dans les territoires étudiés, l'arbre qui représente le mieux la pacification entre les peuples est Faidherbia albida. Dans les campagnes, les parcs à Faidherbia s'étendent au détriment des autres parcs et de la savane. Faidherbia albida est en effet l'arbre le mieux adapté à la nouvelle société agropastorale, à la forte croissance démographique et à l'économie de marché. Son développement est également appuyé par des croyances : il est interdit chez certaines ethnies d'abattre un jeune Faidherbia albida car cela ferait mourir un jeune homme dans le village. 
Figure 11. Faidherbia albida, marqueur du développement du pastoralisme dans une société d'agriculteurs, Barani (Burkina Faso), février 2011

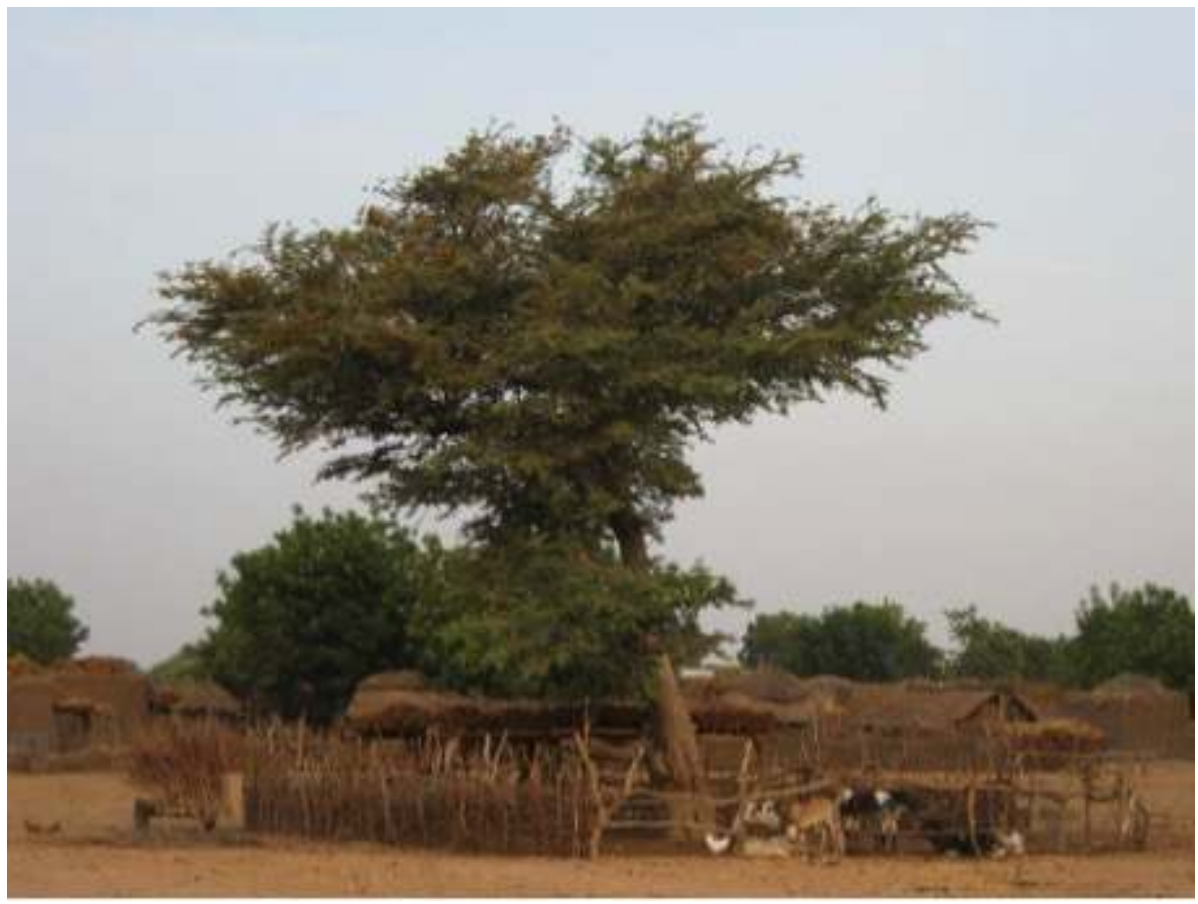

La conjonction de deux facteurs, l'économie de marché (le paysan ne cultive plus seulement pour nourrir sa famille mais également pour la vente), promue par la colonisation européenne, et l'omniprésence du bétail, héritage de l'expansion d'un peuple d'éleveurs, les Peuls, à l'occasion des guerres saintes qui marquent l'Afrique au milieu du XIX $x^{e}$ siècle, va entraîner le développement exponentiel du parc à Faidherbia albida. Cet arbre, du fait de son rythme de feuillaison inverse (dénudé en saison des pluies et couvert de feuilles en saison sèche), crée un véritable microclimat, laissant passer la lumière durant la saison humide, période des cultures, et au contraire protégeant le sol et faisant office de coupe-vent pendant la saison sèche (Roupsard, 1997). Sa litière enrichit le sol et permet ainsi des rendements de cultures pluviales (coton, mil, sorgho) augmentés tout en supprimant la jachère. Les autochtones disent de cet arbre qu'il «ne se nourrit pas de la même eau que les autres». Son système racinaire s'enfonce profondément dans le sol, parfois à plus de 20 mètres, ce qui le rend quasiment indépendant de l'eau de surface et des nutriments des premières strates du sol. La compétition avec les cultures n'existe donc pas. Son feuillage et ses gousses ont une forte valeur nutritive pour le bétail. Celui-ci, en parcourant ces parcs, participe, par ses déjections, à l'enrichissement du sol. De plus, ils ont un long pouvoir de conservation, ce qui permet leur commercialisation sur les marchés à une période où les autres sources de pâturage sont limitées.

Le rôle d'un élevage bovin sédentaire est capital dans la diffusion du Faidherbia albida. Lors du passage dans le système digestif de l'animal, les graines ne sont pas digérées mais la cuticule cireuse est dissoute, processus vital pour la germination de la graine. Une germination qui est d'autant plus facilitée que la graine, débarrassée de son enveloppe protectrice, se retrouve dans les déjections du bétail, engrais naturel. Toutefois, Faidherbia albida ne saurait s'élever seul à l'état d'arbre, il a besoin de l'homme pour dégager une cime et pour être émondé. Ainsi, les parcs de Faidherbia albida actuels sont de véritables constructions que l'on doit aux populations de 
cultivateurs élevant du bétail. Il assure ainsi une pacification entre éleveurs et agriculteurs, dans des régions où les tensions sont nombreuses.

\section{Conclusion}

L'organisation territoriale en Afrique est fortement rythmée par la végétation et notamment les arbres: les arbres des villes, les arbres des champs, les arbres de la savane/forêt. Leur choix et leur répartition sont faits avec une extrême minutie. Ils sont tributaires des modes de vie, des pratiques agricoles, des croyances, des jeux de pouvoirs, des dynamiques urbaines, même dans les délaissés à la dynamique plus sauvage. Cette végétation est le fruit d'un subtil équilibre entre les contraintes environnementales qui impriment la trame de fond du capital végétal (les températures, les précipitations, le type de sol, etc.), les besoins et les moyens des populations, le tout guidé par les habitudes culturelles.

Les exemples du Cameroun et du Burkina Faso illustrent un rapport vital à l'arbre. Les territoires présentés ne peuvent pleinement se comprendre sans s'attarder sur la couverture végétale, qu'elle soit plantée ou pas. Nos travaux montrent que l'arbre, bien plus qu'un simple élément du décor et du quotidien, est aussi un livre ouvert qui retrace l'histoire des territoires et de ses peuples et permet de décrypter les enjeux sociaux et spatiaux actuels.

La fabrication des paysages végétaux est en lien avec des pratiques locales héritées des générations passées et adaptées au contexte actuel. Les grands traits de ces paysages sont le produit du fonctionnement des ethnies avec lequel les stratégies de développement mises en place par les États camerounais et burkinabé doivent composer.

\section{BIBLIOGRAPHIE}

Agulhon, M., Duby, G. (dir.), La Ville de l'âge industriel : le cycle haussmannien. Histoire de la France urbaine, Paris, Éditions du Seuil, 1998.

Ballouche, A., « Forêts versus savanes », dans Chartier, D. et Rodary, E. (dir), Manifeste pour une géographie environnementale, Paris, Presses de Sciences Po, 2016.

Blanchet, A., Gotman, A., L'Entretien, Paris, Armand Colin, 2010.

Boffa, J.-M., Agroforestry parklands in sub-Saharan Africa, Rome, FAO, 1999.

Claval, P., « Champ et perspectives de la géographie culturelle », Géographie et Culture, n 1,1992 , p. 7-38.

Combessie, J.-C., « L'entretien semi-directif », Repères, nº 5, 2007 p. 24-32. 
Devineau, J.-L., Serpantié, G., « Projet SALT : paysages végétaux et systèmes agraires au Burkina Faso ", dans Pouget, M., Caractérisation et suivi des milieux terrestres en régions arides et tropicales, Paris, Orstom, coll. «Colloques et Séminaires », 1991.

Donadieu, P., Sciences du paysage : entre théories et pratiques, Cachan, Tec \& Doc, 2012.

Floret, C., La Jachère en Afrique tropicale : rôles, aménagement, alternatives, Montrouge, Libbey Eurotext, 2001

Garine, É., Moussa, A., Raimond, C., Dounias, E., Kokou, K.,. « Usages alimentaires du parc arboré sélectionné (Duupa, massif de Poli, Nord-Cameroun) », dans Langlois, O., Ressources vivrières et Choix alimentaires dans le Bassin du lac Tchad, Marseille, IRD Éditions, coll. « Colloques et Séminaires ", 2013.

Guengant, J.-P., Seignobos, C., Sodter, F., La Jachère en Afrique tropicale : l'apport des sciences sociales : hommage à Roger Pontanier, Paris, Harmattan, 2006

Herin, « Une géographie des rapports sociaux », Espace géographique, $\mathrm{n}^{\circ}$ 15, 1986, p. 108-110.

Huxley, P.A., Tropical agroforestry, Oxford, Blackwell Science, 1999.

Küster, H., Marwinski, T., Petite Histoire du paysage, Strasbourg Circé, 2013.

Lahuec, J.-P., « Le parc d'un village mossi (Zaongho), du traditionnel au moderne », Cahiers de l'Orstom, Série « Sciences humaines », $\mathrm{n}^{\circ}$ 17, 1980, p. 151-154.

Lespez, L., Ballouche, A., « L'objet, le sensible et leurs trajectoires », Bulletin de l'association de géographes français, $\mathrm{n}^{\circ} 1,2009$, p. 311.

Marcel, O. (dir.), Paysage, mode d'emploi. Pour une histoire des cultures de l'aménagement, Seyssel, Champ Vallon, 2006.

Maydell, H.J. von, Trees and shrubs of the Sahel : their characteristics and uses, Weikersheim, Verlag Josef Margraf, coll. « Scientific Books », 1990.

Mehdi, L., Weber, C., Pietro, F. di, Selmi, W., «Évolution de la place du végétal dans la ville, de l'espace vert a la trame verte ", Vertigo, vol. 12, n², 2012, URL : https://vertigo.revues.org/12670

Méo, G. di, « Une géographie sociale. Entre structures et représentations », Cybergeo, août 2016, URL : http://cybergeo.revues.org/27761

Méo, G. di, « La géographie culturelle : quelle approche sociale ?», Annales de Géographie, 2010, p. 47-66.

Nkamleu, G. B., « La pratique de la jachère en Afrique de l'ouest : Importance comparée du phénomène au Nigeria et au Cameroun ", Sécheresse, n² 2, 1999, p. 2-14.

Pélissier, P., «L'Arbre dans les paysages agraires de l'Afrique Noire », Cahiers de l'Orstom, Série «Sciences humaines », $\mathrm{n}^{\circ} 17,1980, \mathrm{p} .131-136$.

Picouet, M., Sghaier, M., Genin, D., Abaab, A., Guillaume, H., Elloumi, M., Environnement et Sociétés rurales en mutation : approches alternatives, Paris, IRD, coll. « Latitudes », 2004.

Portères, R., «L'ethnobotanique : Place - Objet - Méthode - Philosophie », Journal d'agriculture. tropicale et de botanique appliquée, $\mathrm{n}^{\circ} 8,1961, \mathrm{p} .102-109$.

Roger, A., La Théorie du paysage en France, 1974-1994, Seyssel, Champ vallon, 1995.

Roupsard O, « Écophysiologie et diversité génétique de Faidherbia albida (Del.) A. Chev. (syn. Acacia albida Del.), un arbre à usages multiples d'Afrique semi-aride : fonctionnement hydrique et efficience d'utilisation de l'eau d'arbres adultes en parc agroforestier et de juvéniles en 
conditions semi-contrôlées ", Nancy, Université de Nancy, thèse de doctorat en écophysiologie forestière, 1997

Seignobos, C., «Essai de reconstitution des agrosystèmes et des ressources alimentaires dans les monts Mandara (Cameroun) des premiers siècles de notre ère aux années 1930 », Revue d'ethnoécologie, $\mathrm{n}^{\circ}$ 5, 2014.

Seignobos, C., «L'arbre et la cité dans la zone soudano-sahelienne (les exemples du Tchad et du Cameroun septentrional) ", Revue de géographie du Cameroun, $\mathrm{n}^{\circ}$ 2, 1981, p. 49-52.

Smektala, G., Peltier, R., Sibelet, N., Leroy, M., Manlay, R., Njiti, C. F., Ntoupka, M., Njiemoun, A., Palou, O. et Tapsou, «Parcs agroforestiers sahéliens : de la conservation à l'aménagement », VertigO, vol. 6, $\mathrm{n}^{\circ}$ 2, septembre 2005, URL : http://vertigo.revues.org/4410

Touraine, A., Sociologie de l'action, Paris, Éditions du Seuil, 1965.

Yameogo, G., Yelemou, B., Boussim, I. J., Traore, D., « Gestion du parc agroforestier du terroir de Vipalogo (Burkina Faso) : contribution des ligneux à la satisfaction des besoins des populations », International Journal of Biological and Chemical Sciences, $\mathrm{n}^{\circ}$ 7, 2013, p. 1087-1105.

Yengué, J.-L., « Le rapport au végétal. Pour une lecture renouvelée des territoires », Angers, université Angers, HDR de géographie, 2015.

Yengué, J.-L., Cochonneau, M., « For the development of agroecology at Barani (Burkina Faso) », Journal of Agricultural Science and Technologie, B 3, 2013, p. 545-550.

Yengué, J.-L., « L'arbre des villes, l'arbre des champs. Les processus de construction de paysages au nord du Cameroun ", dans Marcel, O. (dir.), Paysage, mode d'emploi. Pour une histoire des cultures de l'aménagement, Seyssel, Champ Vallon, 2006.

Yengué, J.-L., « Les mécanismes du déboisement et du reboisement dans le nord du Cameroun », Annales de géographie, 2002, p. 27-45.

Yengué, J.-L., Callot, Y., « L'arbre et la ville dans la région de Maroua (Extrême-Nord

Cameroun) », Sécheresse, 2002, p. 155-163.

Yengué, J.-L., « Cartographie du couvert ligneux par télédétection : exemple de la région de Maroua au Nord-Cameroun ", Bulletin de la Société française de photogrammétrie et de télédétection, $\mathrm{n}^{\circ}$ 15, 2001, p. 4-15.

Yengué, J.-L., « L'évolution du couvert ligneux dans l'Extrême-Nord du Cameroun. Utilisation de la photographie aérienne et de l'imagerie satellitaire », Paris, université Paris 1 Panthéon Sorbonne, thèse de doctorat de géographie, 2000.

\section{NOTES}

1. Barani (Pour une aide durable au développement en milieu sahélien au Burkina Faso) 2010-2011, financement des universités de Tours et d'Orléans ;

Biosol (Stimulation biologique des sols et gestion socioéconomique des agrosystèmes au Burkina Faso), 2012-2016, financement de la région Centre-Val de Loire ;

Aura (Agriculture urbaine à Ouagadougou), 2016-2017, financement du réseau des MSH.

2. Il s'agit de langues locales. 


\section{RÉSUMÉS}

Le travail présenté porte sur différents territoires africains situés au Cameroun et au Burkina Faso. L'apparente diversité de ces terrains cache en fait une convergence absolue: la place centrale de l'arbre dans le paysage. Il enrichit les sols, freine l'érosion due au ruissellement, apporte de l'ombre, permet de se chauffer, de se nourrir, de s'éclairer, de se soigner, etc. Il tient également une place importante dans l'imaginaire des populations locales et est un élément essentiel à l'agriculture. Dès lors, l'étude du rapport à l'arbre peut servir à une meilleure connaissance des territoires et permet de proposer une lecture des rapports sociaux. C'est l'objectif de cette contribution. Ce travail montre que l'arbre, bien plus qu'un simple élément du décor et du quotidien, est aussi un livre ouvert dans lequel peut être lue l'histoire des territoires et leurs combinaisons géographiques.

The research presented in this article concerns different regions in Cameroon and Burkina Faso. The apparent diversity of the areas masks a situation of total convergence concerning the central role of the tree in the landscape. The tree enriches the soil, slows down erosion due to run-off water and gives shade. It also provides fuel for heating, cooking and light, and is a source of medicinal products. In addition, the tree occupies an important place in the imagination of local populations and plays an essential role in agriculture. For all of these reasons the study of the relationship to the tree can help gain a better understanding of places and social interactions. This is the objective of this research which shows that the tree is much more than simply a part of the landscape and everyday life : it provides deep insights into the history of regions and their geographical configurations.

\section{INDEX}

Mots-clés : arbre, paysages végétaux, Afrique, usages, Cameroun, Burkina Faso

Keywords : tree, plant landscapes, Africa, customs, Cameroon, Burkina Faso

\section{AUTEUR}

\section{JEAN-LOUIS YENGUÉ}

Jean-Louis Yengué est géographe et maître de conférences HDR, UMR Citeres, CNRS/université de Tours.

yengue[at]univ-tours[dot]fr 